\title{
2D static failure prediction for critical stresses of an Automobile Tire Side Walls using MATLAB PDE Toolbox
}

\author{
Chukwutoo Christopher Ihueze ${ }^{1}$; Chinedum Ogonna Mgbemena ${ }^{2}$; \\ A. R. Ravindranatha Menon ${ }^{3}$ \\ ${ }^{I}$ Department of Industrial Production Engineering, Nnamdi Azikiwe University, Awka, Nigeria \\ ${ }^{2}$ Department of Mechanical Engineering, Federal University of Petroleum Resources, Effurun, Nigeria \\ ${ }^{3}$ Functional Materials, National Institute for Interdisciplinary Science and Technology, Thiruvananthapuram, \\ 695019, Kerala, India
}

\begin{abstract}
Critical stresses responsible for static failure of tire sidewalls were predicted using MATLAB PDE Toolbox.A model ofthe tire sidewall was developed using the PDE toolbox GUI draw tool and the parameters characterizing the linear isotropic elastic behaviour of rubber vulcanizates were established. The study was developed on maximum inflation pressure of 0.2206MPa and results obtained from the study indicates that the tire material developed from Natural Rubber/Rubber Seed oil modified kaolin composite are acceptable under static analysis with critical stress values of 5-25MPa obtained around the tire sidewall which are higher than the applied maximum inflation pressure. The maximum shear stress obtained is $15 \mathrm{MPa}$ at the periphery of the sidewall while the values obtain within the sidewalls ranges from -10 to $10 M P a$. The $2 D$ FE model computations for static loading of the tire gave reasonably good prediction of the critical stresses responsible for failure and proved that the new tire material developed to be functional for tire sidewall applications.
\end{abstract}

Keywords:Finite Element Analysis, Kaolin, MATLAB, Natural Rubber, Stress, Tire

\section{Introduction}

Automobile Tires provide traction between the vehicle and the road while providing a flexible cushion that absorbs shock. The tire is a very complex and complicated toroid composite structure made up mostly of rubber, textile-cords and steel-cords. A tire is composed of several layers which are known as plies. Each layer is a different type of fabric.

The tire sidewall is just one of various parts that constitute the standard tire. It bridges between the tread and bead. The sidewall is made of rubber but reinforced with fabric or steel cords that provide for tensile strength and flexibility. The tire sidewalls contains air pressure and transmits the torque applied by the drive axle to the tread to create traction but supports little of the weight of the vehicle, as evidenced from the total collapse of the tire when punctured. It performs the functions of preventing air from leaking from the tire; keeping the body plies protected; provision of lateral stability to the tire. The sidewall are molded with manufacturer-specific detail, government mandated warning labels, and other consumer information.

Virtually all FE simulations performed on tires emanating from literatures published are on static analysis. Static analysis as applied to automobile tire designs are performed in other to determine the load carrying capacity of tires, robustness of the finite element analysis, sophistication of the software used, the level of mesh refinement with respect to the number of nodes and elements used. The most important reason for implementation of the static analysis for tires is for predicting the possible points of failure at critical points in the structure. A static FEA may be employed in the analysis of the first function of the tire and the support of load, in order to predict vertical stiffness with respect to the shape of load-deflection curve and also to analyze footprint shape and stress distribution [1-3].

Lee et al. [4] performed a non-linear static and transient FEA analysis of a tire model by simulating the radial and lateral static stiffness test conditions, conducting dynamic free-drop test and determining the rolling cornering stiffness, but their analysis didn't focused on the bed-rim interaction.

Tiberiu et al. [5] conducted a study on the static and transient analysis of radial tires using ANSYS. Their paper gave more insights on modeling and simulation of the static and dynamic behavior of radial tires for civil emergency vehicles or military armored vehicles with their results compared with the imprint of the tire on the road surface.

Chatterjee and Ranjan [6] did a study on finite element modeling of tire using ANSYS to analyze the free vibration of radial pneumatic tires. In their work, they investigated the natural frequencies and mode shapes of pneumatic tires.

Molisani et al. [7] investigated the tire modeled as an annular cylindrical shell in which the outside shell is flexible, the tire sidewalls and wheel are assumed rigid and considered the analytical solution of the eigenproblems, both the tire structure and cavity acoustic responses were expanded in terms of their eigenfunctions. 
Yan et al. [8] performed a research on the analysis of a radial tire using a Finite Element Method. In their study, a new mathematical model on extension propagation of interface crack in complex composite structures was developed.

Mohsenimanesh et al. [9] investigated the stress analysis of a tractor tire using Finite Element Analysis. In their study, the modelling process was based on the 3D pressure fields obtained from the non-linear static stress analysis of finite element tire model, which considers the structural geometry, the anisotropic material properties of multiple layers and the nearly incompressible property of the tread rubber block.

Ghoreishy et al. [10] developed a 3D finite element model for the modeling of a P155/65R13 steelbelted tire under inflation pressure, vertical static (footprint) load, and steady state rolling. The model was created for the ABAQUS/Standard code and used to carry out a series of parametric studies [11].

The present study is centered on static FEA of a P195/55 R16 85H radial tire sidewall which is an existing wheel configuration. The FEA was performed using MATLAB PDE Toolbox on the tire sidewall to determine the functionality of a new tire material developed from Natural Rubber/Rubber Seed Oil modified kaolin composite, in order to find improved design solutions and predict critical stress points on the sidewalls where catastrophic failure may likely occur in other to plan for containment.

\subsection{Materials}

\section{Methodology}

The materials used in this study are BCK grade Kaolin obtained from English Indian Clays Ltd. Thiruvananthapuram, Kerala, India; Rubber seed oil (RSO) and Natural Rubber (RSS V grade) which were provided by NIIST, CSIR, Thiruvananthapuram, India; Laboratory grades of Sodium hydroxide (MERCK) and Hydrazine hydrate (FINNAR) obtained from local suppliers in Thiruvananthapuram, Kerala, India.

\subsubsection{Conversion of RSO into its sodium salt}

The Sodium salt of rubber seed oil (Na-RSO) was prepared stoichiometrically by mixing $33 \mathrm{~mL}$ of RSO with $100 \mathrm{~mL}$ of $20 \% \mathrm{NaOH}$ solution in an ice bath along with continuous stirring for about 12 hours. The resulting mixture was kept for a further period of 24 hours to cure. The final $\mathrm{pH}$ of the resulting solution was adjusted to 8-9. The Na-RSO was washed with water to remove excess of $\mathrm{NaOH}$. Excess water was removed by heating at a temperature $\sim 120^{\circ} \mathrm{C}$ for few hours in a hot air oven, and the product was powdered.

\subsubsection{The Synthesis of RSO modified kaolin}

$98 \mathrm{~g}$ of Kaolin was slowly added to a mixture-containing $100 \mathrm{~g}$ of Na-RSO, $70 \mathrm{~mL}$ hydrazine hydrate with vigorous stirring at $20^{\circ} \mathrm{C}$. The mixture was homogenized using an Art-MICCRA D-8 (Germany) homogenizer, and the sample was dried using a freeze drier [HetroTrap-CT60e, JOUAN].

\subsubsection{Preparation of Rubber Vulcanizates}

Natural Rubber used in this study were initially flattened for about 5-10 min in an open two-roll mill at temperatures $\sim 30-40^{\circ} \mathrm{C}$. The rubbers were combined and mixed with accelerants, activators, the RSO modified kaolin, softeners and lastly, Sulphur was added. The mixtures were plasticized for about $15 \mathrm{~min}$ and thin-passed several times at $90^{\circ} \mathrm{C}$. The obtained vulcanizates were allowed to cure. The Rubber vulcanizates sheets were prepared by compression molding of the mixes at $140^{\circ} \mathrm{C}$ for $10 \mathrm{~min}$ on an electrically heated, semi-automatic hydraulic press (MODEL INDUDYOG DS-SD-HMP/25) at 3 torr pressure (i.e. $400 \mathrm{~Pa}$ ) and curing takes place at the same temperature and pressure. Replicated samples of the vulcanizates were prepared by melt blending and subjected to uniaxial tensile tests to ascertain their material properties as explained in the recipe of Table 1 .

Table 1 Recipe of the Rubber Compounds

\begin{tabular}{|lc|}
\hline Ingredients (phr) & MRK \\
\hline Natural Rubber & 100 \\
Zinc Oxide & 5 \\
Stearic Acid & 2 \\
MRK & 10 \\
MBT & 2 \\
Sulphur & 2 \\
\hline
\end{tabular}

MRK is the RSO-modified Kaolin; MBT is Mercapto Benzothiazole.

\subsection{Static Failure Analysis for static loading}

The failure analysis for static loading can be summarized for a two dimensional planar material with the following sets of equations.

The stress tensor for a $2 \mathrm{D}$ case is expressed as: 
$\left[\begin{array}{ll}\sigma_{x x} & \tau_{x y} \\ \tau_{y x} & \sigma_{y y}\end{array}\right]$

Where $\sigma_{x x}, \sigma_{y y}$ are the normal stresses; $\tau_{y x}, \tau_{x y}$ are the shear stresses that act in the direction parallel to $\mathrm{x}$ and $\mathrm{y}$ axis.

The strain for $2 \mathrm{D}$ case is expressed in matrix form as:

$\left[\begin{array}{ll}\varepsilon_{x x} & \varepsilon_{x y} \\ \varepsilon_{y x} & \varepsilon_{y y}\end{array}\right]$

Where $\varepsilon$ stands for shear strain as represented by their dual subscripts.

For a $2 \mathrm{D}$ stress state, the principal stress is expressed as:

$$
\begin{aligned}
& \sigma_{1}, \sigma_{3}=\frac{\sigma_{x}+\sigma_{y}}{2} \pm \sqrt{\left(\frac{\sigma_{x}-\sigma_{y}}{2}\right)^{2}+\tau_{x y}^{2}} \\
& \sigma_{2}=0 \\
& \tau_{\max }=\tau_{13}=\frac{\left|\sigma_{1}-\sigma_{3}\right|}{2}
\end{aligned}
$$

Von Mises Effective stress as implemented for a 2D case is either expressed as:

$$
\begin{aligned}
& \sigma^{\prime}=\sqrt{\sigma_{1}^{2}-\sigma_{1} \sigma_{3}+\sigma_{3}^{2}} \\
& \text { Or } \\
& \sigma^{\prime}=\sqrt{\sigma_{x}^{2}+\sigma_{y}^{2}-\sigma_{x} \sigma_{y}+3 \tau_{x y}^{2}}
\end{aligned}
$$

\subsection{FE implementation using MATLAB PDE Toolbox}

The FEA is implemented in MATLAB PDE Toolbox using the elliptical PDE equation under dirichlet boundary condition.

$-\nabla \cdot(c \nabla u)+a u=$ fin $\Omega$

Where $\nabla$ is the vector $(\partial / \partial x, \partial / \partial y)$, and $c$ is a 2-by-2 matrix function on $\Omega$. The bounded planar domain of interest $c, a$, and $f$ can be complex valued functions of $\mathrm{x}$ and $\mathrm{y}$.

The dirichlet boundary condition is specified as $h u=r$ on the boundary, $\partial \Omega$.

For a $2 \mathrm{D}$ case, the Dirichlet boundary condition is expressed as:

$h_{11} u_{1}+h_{12} u_{2}=r_{1}$

$h_{21} u_{1}+h_{22} u_{2}=r_{2}$

For nonlinear elliptic PDE, the nonlinear Newton solver is available for solutions, it is expressed as:

$-\nabla \cdot(c(u) \nabla u)+a(u) u=f(u)$

Where the coefficients defining $c, a$, and $f$ can be functions of $\mathrm{x}, \mathrm{y}$, and the unknown solution, $\mathrm{u}$. All the solvers can address the PDE system with multiple dependent variables

$$
\begin{aligned}
& -\nabla \cdot\left(c_{11} \nabla u\right)-\nabla \cdot\left(c_{12} \nabla v\right)+a_{11} u+a_{12} v=f_{1} \\
& -\nabla \cdot\left(c_{21} \nabla u\right)-\nabla \cdot\left(c_{22} \nabla v\right)+a_{21} u+a_{22} v=f_{2}
\end{aligned}
$$

The PDE Toolbox can handle all 2D problems and it also provides an adaptive mesh refinement algorithm for elliptic and nonlinear elliptic PDE problems. In this work, MATLAB PDE Tool for elliptical equation under dirichlet boundary condition was used to solve for structural mechanics plane stresses and subsequently the yield stress distribution.

Following the experimental data generated from stress-strain analysis of the material to establish the material property as shown in Table 2 and figure 1. The FEA was performed to predict the critical stress points using elliptical PDE mode on MATLAB.

The followingassumptions were made in this study:

- Linear isotropic elastic stress-strain behaviour is assumed for the tire material investigated.

- Only the 2D view of sidewall was considered. 
- The analysis was performed for the Natural Rubber/Rubber seed oil modified kaolin composites used as tire material.

- The tire thread and other reinforcements were not considered.

- The maximum inflation pressure of 220631.82 Pa for vehicle tire size of P195/55 R16 85H was used in the analysis.

- The applied inflation pressure is assumed to be deformation-dependent so that the pressure always remains normal to the tire internal surface.

- The tire contact surface is assumed frictionless surface in this analysis.

The ISO Metric tire designation for the tire size P195/55 R16 85H investigated is as follows:

P: Passenger tire, 195: Nominal Section Width (measured in millimeters), 55: Aspect Ratio (ratio of the height of the tire's cross section, to its width), R: Carcass construction (R for Radial), 16: Rim Diameter (measured in inches), 82: Load Rating (Service Description, from Load Index table $=515 \mathrm{Kg}$ ), H: Speed Rating (Service Description, from Speed Symbol table $=210 \mathrm{~km} / \mathrm{h}$ ).

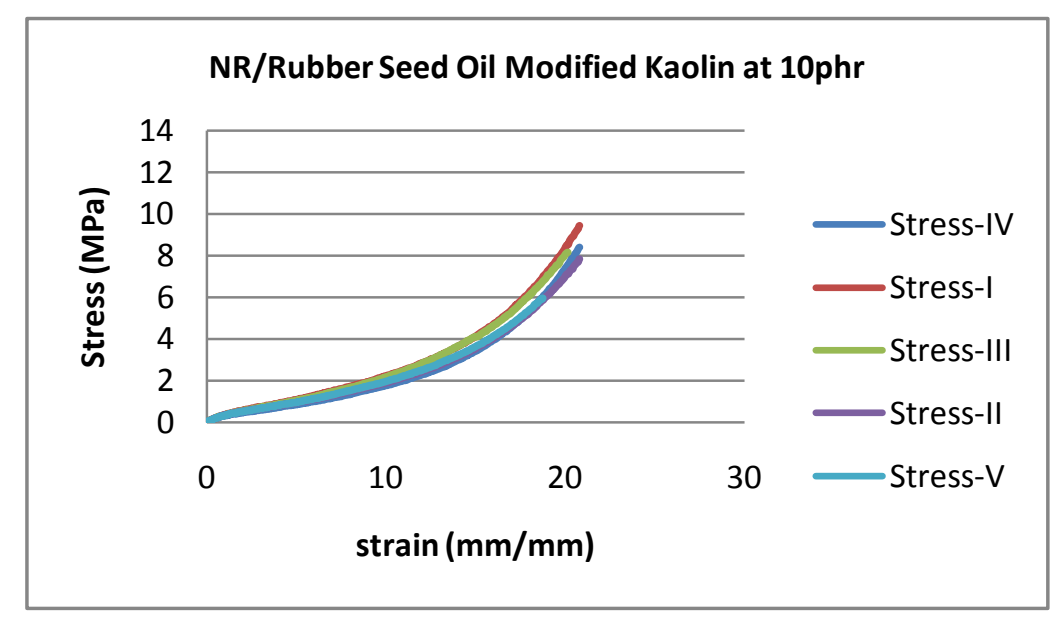

Fig 1 Stress-Strain plot of NR/Rubber Seed Oil Modified Kaolin at 10phr

Table 2 Material Properties of Natural Rubber/Organomodified kaolin Composites

\begin{tabular}{|ll|}
\hline Property & Value \\
Elastic modulus, E & $20700 \mathrm{~Pa}$ \\
Density, $\rho$ & $1186 \mathrm{~kg} / \mathrm{m}^{3}$ \\
Poissons ratio, v & 0.47 \\
Inflation pressure, $P_{i}$ & $220631.82 \mathrm{~Pa}$ \\
\hline
\end{tabular}

Table 3 Tire geometry specification for a P195/55 R16 85H

\begin{tabular}{|ll|}
\hline Specification & Dimension(mm) \\
Sectional width & 195 \\
Rim diameter & 410 \\
Outer diameter & 624 \\
\hline
\end{tabular}

\subsection{Finite Element Tire sidewall Model}

The main attraction of PDE Toolbox is the simplicity and ease in performing 2D analysis which involves the calculation of yield stresses obtained as von Mises stresses, orthogonal stresses and etc. with shorter computation time. The PDE Toolbox has a PDE solver that utilizes the Finite Element Method (FEM) for problems defined on bounded domains in the plane and follows the following simple steps for solving equations:

$>$ Draw the domain using the draw tool in the Graphical User Interface (GUI)

$>$ Set up the appropriate boundary conditions.

$>$ Define the governing equations.

$>$ Generate the mesh

$>$ Discretize the equations and solve on the mesh.

$>$ Plot the results.

The GUI helps the user get through with these steps with ease. 


\subsubsection{Tire sidewall Geometry}

The tire sidewall material developed is shown to be hyperelastic with very large deformation upon loading as exemplified in Figure 1. The 2D model of the tire sidewall was developed from MATLAB PDE Toolbox GUI draw tool. Figure 2 depicts a 2D physical model for the tire sidewall which takes into consideration all the details on the tire size as shown in table 3. The 2D meshed tire sidewall model has 1004 nodes and 1792 triangle elements.

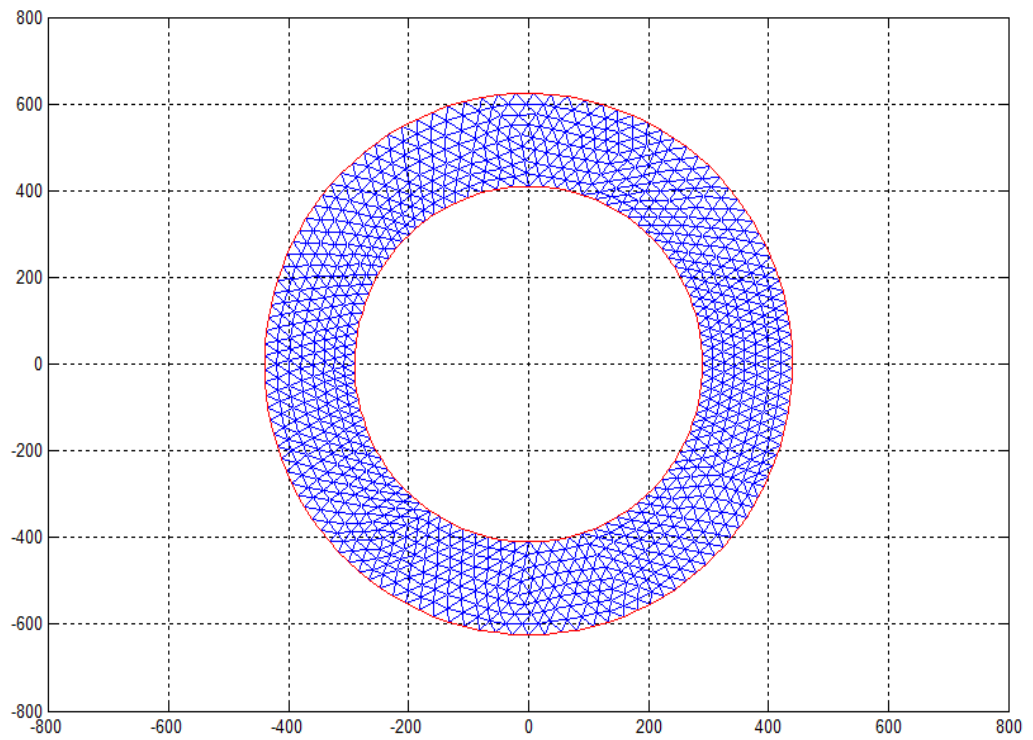

Fig 2 Tire sidewall mesh showing 1004 nodes and 1792 triangle elements

\subsubsection{Boundary Conditions}

In line with the dirichlet boundary condition, uniform pressure of $220631.82 \mathrm{~Pa}$ which is the maximum inflation pressure for the tire size investigated was applied on the internal surface of the tire.

\subsubsection{Numerical Analyses}

The analyses were performed using MATLAB Partial Differential Equation Toolbox 1.2 (pdetool) based on structural mechanics, plane stress analysis. The inflation analysis was performed on the 2D model.

\subsection{Total displacement of Tire Sidewall}

\section{Results And Discussions}

The deformation of the 2D tire sidewall model and the magnitude of shape displacements are shown in Fig. 3. The predominant magnitude of displacement within the sidewalls ranges from $0 \mathrm{~mm}$ for lower limits around the sidewall periphery (rim area and the thread area) and up to $80000 \mathrm{~mm}$ for upper limits within the sidewalls. This observation may be due to increased reinforcement within the peripherals of sidewalls to provide for tensile strength, flexibility and traction.

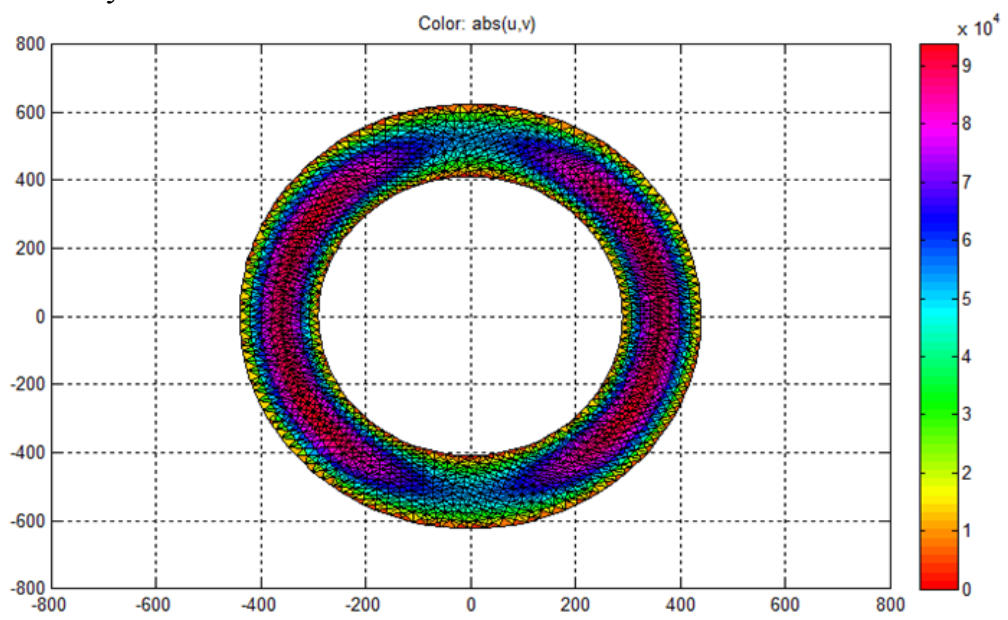

Fig 3 Total displacement of Tire sidewall 


\section{2 von-Mises Hencky stress of the tire sidewall}

The von-Mises-Henky stresses of the tire sidewall for maximum inflation tire is shown in Figure 4 to be within the range of $25 \mathrm{MPa}$ for maximum inflation pressure of $0.22 \mathrm{MPa}$. This critical stress value was found to occur around the rim area and the thread areas respectively. At the sidewall, the critical stresses obtained are within the range of 5-20 $\mathrm{MPa}$.

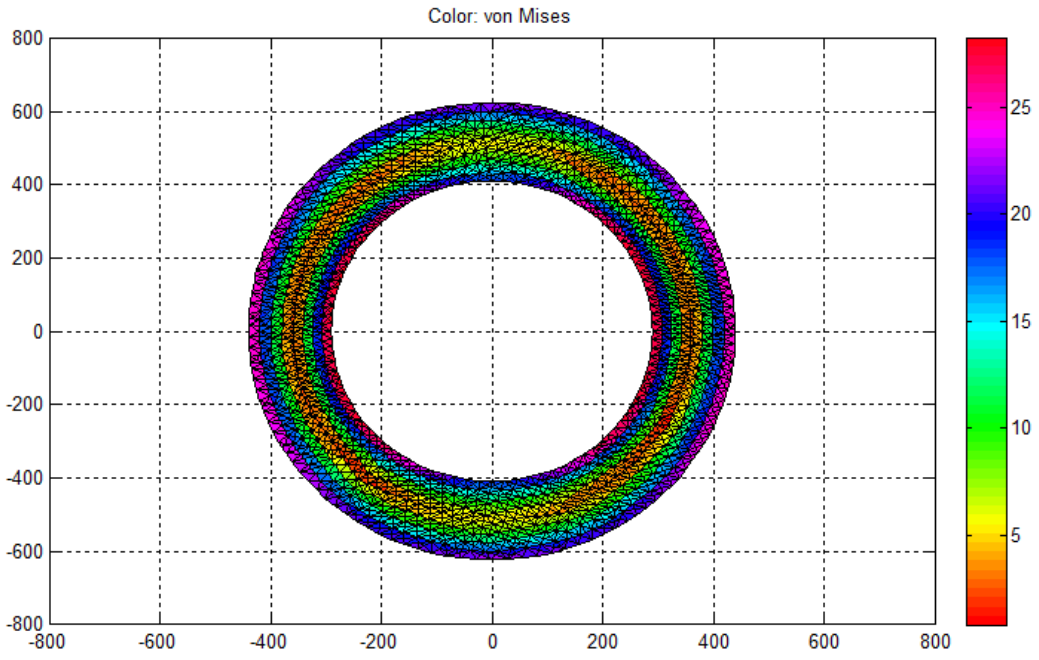

Fig 4 The von Mises stress of the tire sidewall

\subsection{Shear stresses of the tire sidewall}

The most significant characteristic of the finite element analysis of a tire under static load is to analyze the state of stress and strain at different locations of the tire carcass. The shear stress of the tire sidewall connotes the tire endurance under external load and is an important property employed in tire design and construction. In this study, the maximum shear stress obtained is $15 \mathrm{MPa}$ and it is situated near the tire thread area and the minimum value of $-15 \mathrm{MPa}$ around the rim. However, the sidewall recorded values from -10 to $10 \mathrm{MPa}$.

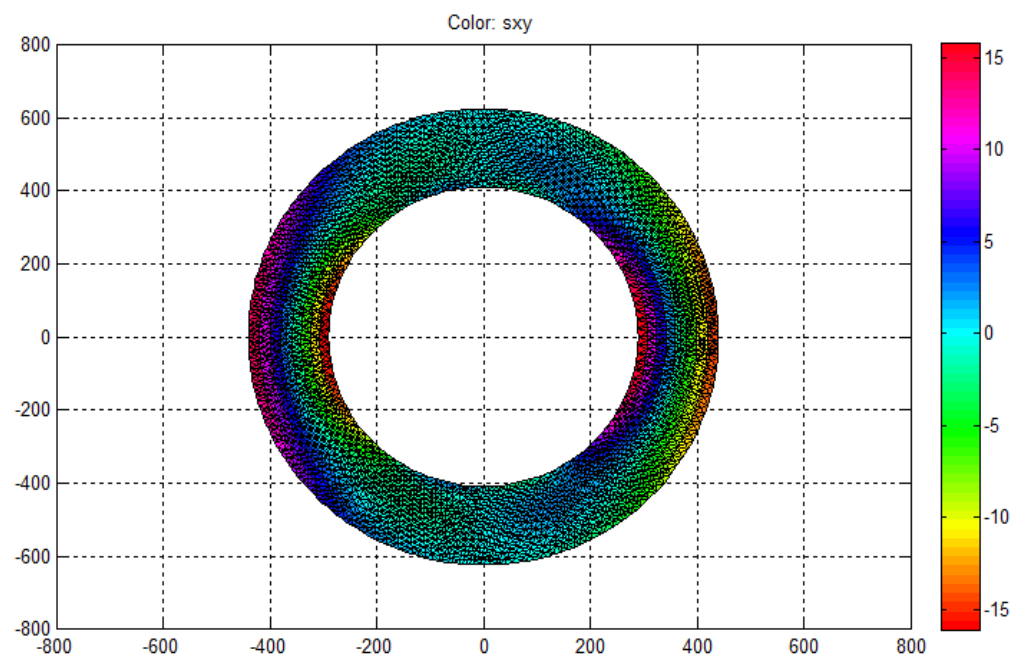

Fig 5 Shear stresses of the tire sidewall

\subsection{Shear strain of the tire sidewall}

The shear strain result of the tire sidewall is associated with durability and fatigue life of the sidewall. Minimum values obtained will amount to tires with better endurance (Ghoreishy, 2001). In the present study, the value obtained in the periphery of the sidewall is 2000 while the values obtained within the sidewall were found to be from 0 to 1000 irrespective of its direction. The result obtained is acceptable for the tire material developed. 


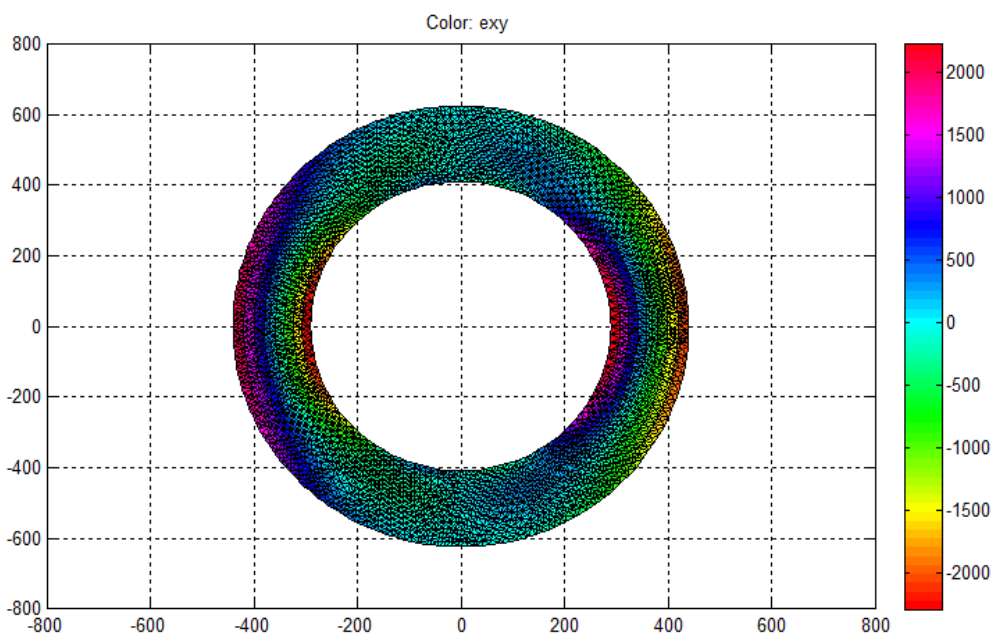

Fig 8 Shear strain of the tire sidewall

\section{Conclusions}

Finite element analysis was implemented using MATLAB Partial Differential Equation Toolbox to investigate the functionality of a P195/55 R16 $85 \mathrm{H}$ pneumatic tire developed from Natural Rubber/Organomodified kaolin subjected to static loading and predict the critical stress points on the tire sidewall. From the present study, the following conclusions were made:

1. MATLAB PDE Toolbox can effectively be employed in Static FE Analysis as the results obtained offered a good analytical evaluation of the tire material studied and is recommended for the use in tire design.

2. The 2D tire model allowed simulating tire inflation with reduced computational efforts and resources.

3. The maximum inflation provided the total deformations of the 2D tire sidewall investigated.

4. The critical stress points from the von Mises result are located around the rim area and tire thread area with value of $25 \mathrm{MPa}$.

5. von Mises stresses for the sidewall were evaluated to be in the range $5 \mathrm{MPa}-25 \mathrm{MPa}$

\section{Acknowledgements}

The authors thank Dr. Suresh Das, Director, National Institute for Interdisciplinary Science and Technology, Thiruvananthapuram, Kerala, India for providing the facilities for the work; Centre for International Co-operation in Science for DST-RTFDCS Fellowship to one of the authors.

\section{References}

[1]. N. Korunović , M. Trajanović, M., Stojković. "FEA of tyres subjected to static loading", Journal of the Serbian Society for Computational Mechanics, Vol. 1, No. 1, 2007.

[2]. G.R. Potts, 'The Tire as a Vehicle Component', A Course Presented by the Department of Textiles at UMIST and University of Acron, 1979.

[3]. R.A. Ridha, M. Theves, "Advances in Tire Mechanics", Rapra Rewiew Report, Vol. 7, No. 5, RAPRA Technology ltd., 1994.

[4]. C. Lee, J. Kim, J. Hallquist, Y. Zhang, et al. "Validation of a FEA Tire Model for Vehicle Dynamic Analysis and Full Vehicle Real Time Proving Ground Simulations," SAE Technical Paper 971100, 1997.

[5]. G. Tiberiu, C. Florina,P. Cristina, "Static and Transient Analysis of Radial Tires Using ANSYS", Recent Advances in Industrial and Manufacturing Technologies, no date.

[6]. A. Chatterjee, V. Ranjan."Free Vibration Analysis of Radial Pneumatic Tire Using FEM", International Journal of Emerging Technology and Advanced Engineering, Volume 2, Issue 8, August 2012.

[7]. L.R. Molisani, R.A. Burdisso, D. Tsihlas, "A coupled tire structure/acoustic cavity model", International Journal of Solids and Structures 40, 5125-5138,2003.

[8]. X. Yan, Y. Wang, X. Feng, Study for the endurance of radial truck tires with finite element modeling, Mathematics and Comput ers in Simulation, 471-488, 2002.

[9]. A. Mohseninmanesh, S.M. Ward, M.D. Gilchrist, Stress analysis of a multi-laminated tractor tyre using non-linear 3D finite element analysis. Material and Design. 30: 1124-1132, 2009.

[10]. M.H.R. Ghoreishy, M. Malekzadeh, H.A. Rahimi, Parametric Study on the Steady State Rolling Behavior of a Steel-Belted Radial Tire, Iranian Polymer Journal, 16, pp. 539-548., 2007.

[11]. M.H.R.Ghoreishy, Finite Element Analysis of a 6 .45-14 Bias Tire under Contact Load, Iranian Polymer Journal, vol. 10, 1, pp.48$52 ., 2007$. 